\title{
An Analysis of the Paratext in Graham Sanders's Six Records of a Life Adrift: Features and Functions
}

\author{
Fangwen PENG* and Linxin LIANG**
}

\begin{abstract}
As a classic piece of Chinese literature, Shen Fu's (1763-1825) - a famous writer and literary figure of the Qing dynasty_Fu Sheng Liu Ji (Six Records of a Life Adrift) has been translated into more than ten languages. To date, it has had four English versions, and enjoys a favorable reception in the English-speaking world. By analyzing the paratext (including footnotes, the introduction, the note on the translation, the title, illustrations, and other elements) of the latest English version by Canadian scholar-translator Graham Sanders, this paper intends to identify the features of the paratext in terms of spatial distribution, category distribution, and detail density, as well as its functions of facilitating the reader's reception of the work, explaining unfamiliar cultural concepts, highlighting the translator's role, and so on. The paper finds that the paratext contributes to a clear and comprehensive understanding of the translated work, and it, as the peritextual and epitextual ties, plays an important role in the translation, publication, and international reception of the translated work, which affect the canonization of Chinese literature in the English-speaking world. Hopefully, this paper will provide a valuable reference for translation theory and practice as well as relevant research in the future.
\end{abstract}

Keywords: Fu Sheng Liu Ji; translation; paratext; features; functions

\section{Introduction}

Fu Sheng Liu Ji (Six Records of a Life Adrift) is a well-celebrated piece of classic Chinese literature, depicting the true love story of Shen Fu and his wife Chen Yun. The text is praised for its literary beauty, cultural aesthetics, and authentic records of civil life during the late Qing dynasty. The work has been translated into more than ten languages, including four English versions by Lin Yutang (1939), Shirley Black (1960), Leonard Pratt and Chiang

\footnotetext{
* Master's student at the University of Bath.

E-mail: pengfangwen0810@gmail.com; ORCID ID: https://orcid.org/0000-0002-8559-2070.

** Associate professor at Huazhong University of Science and Technology, Wuhan.

Corresponding author. E-mail: linxinliang@hust.edu.cn; ORCID ID: https://orcid.org/0000-0003-1692-2563.

(Received 30 September 2021; accepted 26 November 2021)
} 
transLogos 2021 Vol 4 Issue 2

Peng, Fangwen, and Linxin Liang, pp. 1-26

trans Dogos

A Translation Studies Journal

An Analysis of the Paratext in Graham Sanders's

Six Records of a Life Adrift: Features and Functions

(C) Diye Global Communications diye.com.tr|diye@diye.com.tr

Su-hui (1983), and Graham Sanders (2011).

Six Records of a Life Adrift is an up-to-date version by Canadian sinologist Graham Sanders. It includes some additional materials, such as acknowledgments, an introduction, a note on the translation, maps, footnotes, a chronology, a list of Shen Fu's associates and his family tree, and a list of the historical figures he mentions, which are well-known examples of paratext. This paper aims to examine the paratextual elements in this version to understand the features and functions of paratext in translated works and their publication and international circulation.

\section{Literature Review}

\subsection{Previous Studies on Fu Sheng Liu Ji}

Fu Sheng Liu Ji has been attracting considerable interest from the academic community at home and abroad. For example, the China National Knowledge Infrastructure database reveals that Dong Hui (2002) summarized the features of Lin's translation, mentioning exemplary strategies for translation from classic Chinese into English; Li Yuliang (2005) discussed the merits of Lin's translation, with special attention to what was excluded, including the loss of some conventional connotations, the absence of historical and cultural content, and a deviation from the original style; and Deng Yan (2010) examined the translation strategy used in Pratt and Chiang's version, holding that this version takes readers' anticipation and reception ability into consideration and maintains the features of Chinese culture. Furthermore, the online Web of Science database indicates that Lu Fang (2010) compared Lin's, Black's, and Pratt and Chiang's versions, and examined them on the cultural, aesthetic, and linguistic levels; Guan Xingzhong (2014) explored the translation variations in Lin's translation through analyzing the translator's preface; and Liang Linxin and Xu Mingwu (2018) delved into the four versions' respective translation journeys, as well as their publication routes and international circulations. 
transLogos 2021 Vol 4 Issue 2

Peng, Fangwen, and Linxin Liang, pp. 1-26

trans Logos

A Translation Studies Journal

An Analysis of the Paratext in Graham Sanders's

Six Records of a Life Adrift: Features and Functions

(C) Diye Global Communications diye.com.tr|diye@diye.com.tr

It can be seen that since Sanders's translation is relatively recent, it has not attracted much attention in relevant studies. Furthermore, Sanders $(2011$, vi) acknowledged that he referenced previous translators' works and that his main goal in presenting a new translation was to correct previous errors, add more detailed annotations, and introduce a more colloquial and flowing style of English. It is therefore a suitable occasion for discussing the value and role of this new English version.

\subsection{Previous Studies on Paratext in the Field of Translation Studies}

Paratext, as proposed by Gérard Genette (1997), refers to various verbal and nonverbal materials accompanying a text, such as an introduction, notes, dedications, a preface, a foreword, figures, the book jacket, pictures, epigraphs, and blurbs. Paratexts serve as thresholds, that is "the literary and printerly conventions that mediate between the world of publishing and the world of the text" (Genette 1997, xvii). As such, paratext exerts a considerable influence on the reader's reception of a text, including a translated text (Luo and Zhang 2018, 596).

Until now, a growing body of literature in the field of translation studies has shared a focus on the paratext of translated works. The following presents some of the most relevant literature. The China National Knowledge Infrastructure database reveals that Wei Jiahai (2017) examined the annotations in the English translation of Chu Ci (The Songs of the South) to identify their functions as a cultural narration of the translated Chinese culture; Huang Peixi (2018) delved into the paratext and cultural construction in Ilza Veith's English translation of Huangdi Neijing Suwen (The Yellow Emperor's Classic of Internal Medicine); and Yu Yanghuan and Dong Yan (2020) made a paratextual interpretation of Cyril Birch's English translation of Mu Dan Ting (The Peony Pavilion) to categorize two kinds of paratext in translation studies, namely intentional paratext and contextual paratext, and analyze their connotations and functions. Moreover, the online Web of Science database indicates that Brigid Maher (2016) explored the way Italian crime fiction is presented to prospective 
transLogos 2021 Vol 4 Issue 2

Peng, Fangwen, and Linxin Liang, pp. 1-26

trans Dogos

A Translation Studies Journal

An Analysis of the Paratext in Graham Sanders's

Six Records of a Life Adrift: Features and Functions

(C) Diye Global Communications diye.com.tr|diye@diye.com.tr

Anglophone readers through paratextual binding, namely titles, cover images, and blurbs; Luo Tian and Zhang Meifang (2018) conducted a descriptive case study of Lionel Giles's English translation of Sun Zi Bing Fa (The Art of War) and found that there are three methods, namely supplementation, comparison, and evaluation, used in the paratext to reconstruct a respectable Chinese strategic culture; and Sang-Bin Lee (2020) examined the misogynistic paratext of translations published in South Korea to show how gender ideology could affect the reception and revision of paratext and how closely translations and paratext could be interrelated in a feminist context.

In sum, the paratext of translated works, as a dynamic and rich translation phenomenon, is a gateway that connects the author, the translator, the readers, and the publisher. An analysis of paratextual elements in a literary work or its translated version will reveal features and functions of paratext, the motivation for its publication, the identity of its readership, its connection with the source culture, the translator's view and strategies, and other valuable discoveries. Furthermore, the peritext reveals the relationship between the author, the translator, and the publisher, clarifies the motivation, identifies the target readership, and creates a picture of the temporal and cultural background for translation and publication (Xiao 2011). Therefore, the paratext is inalienable from a text, as it provides essential supplementary information for the reader, the public, and society.

\section{Research Design}

\subsection{Research Questions}

In this paper, a thorough analysis of the features and functions in the paratext of Six Records of a Life Adrift will be presented. By examining each element of the peritext, it will become evident how such elements contributed to the translation. This paper will answer the following questions:

(1) What are the paratextual elements of Six Records of a Life Adrift?

(2) What are the features and functions of the paratext? 
transLogos 2021 Vol 4 Issue 2

Peng, Fangwen, and Linxin Liang, pp. 1-26

trans Dogos

A Translation Studies Journal

An Analysis of the Paratext in Graham Sanders's

Six Records of a Life Adrift: Features and Functions

(C) Diye Global Communications diye.com.tr|diye@diye.com.tr

(3) How can this paper supplement previous studies and contribute to future research?

\subsection{Research Objectives and Methods}

The paratext of Six Records of a Life Adrift analyzed in this paper is characterized as peritext, which includes the acknowledgments, the introduction, the note on the translation, the maps, the footnotes, the chronology, the list of associates and the family tree, the historical figures mentioned, the index, the illustrations, the title and record titles, the cover, and more. The analysis focused on the footnotes, the introduction, and the note on the translation, which take up a lot of space.

To understand how each element functions, content analysis, quantitative analysis, and qualitative analysis are conducted as appropriate. Content analysis involves analyzing the manifest content of the text through classification or evaluation in order to ascertain its probable effect. Quantitative and qualitative analyses entail collecting and interpreting data to ascertain the text's latent features and functions.

The footnotes - 151 in total-occupy the largest part of the peritext in this work; therefore, footnotes will be analyzed both quantitatively and qualitatively. They are categorized into four classes and seven subclasses, serving to clarify literary figures, literary works, historical events, geological information, biological backgrounds, cultural phenomena, ideological differences, etc. The features and functions of these different categories are listed in charts, which will be interpreted qualitatively. In the introduction, Sanders summarizes the plot of the four existing records and tells Shen Fu and Chen Yun's life story. He presents a relatively objective yet slightly sympathetic perspective on Shen. In terms of the note on the translation, he explains the conversion systems used for the calendar, measurements, and currency, and offers other necessary information that was altered during translation. This short text outlines Sanders's strategies and emphases while translating. 
transLogos 2021 Vol 4 Issue 2

Peng, Fangwen, and Linxin Liang, pp. 1-26

\section{Results and Discussion}

\subsection{The Features and Functions of the Footnotes}

Genette (1997) divided notes into four categories: discursive, allographic, auctorial, and fictional. Discursive notes were further divided into three subcategories: original notes, later notes, and delayed notes. Sanders's footnotes have features that fall into multiple categories. According to Genette (1997), the functions of these notes vary, but most importantly, they provide definitions or explanations; disclose figurative meanings; identify quotations or translations; indicate sources or supporting evidence; clarify ignored uncertainties or complexities; put forward additional arguments; or make statements to forestall potential objections.

The features of the footnotes correspond to their functions. In Sanders's version, the features and functions of the footnotes can be summarized as follows:

(1) The footnotes are very detailed and sound in providing annotated information.

(2) The footnotes focus on cultural content, but they also cover various other topics.

(3) The original text highlighted by the footnotes can be proper names, locations, events, quotations, allusions, and other ambiguous concepts.

(4) Most footnotes have multiple functions.

The features and functions of the footnotes in Sanders's version will be illustrated and exemplified in the following pages. The 151 footnotes in Sanders's version include explanations about various subjects, from literary figures and works to historical figures and events, and cultural concepts and phenomena. Therefore, the footnotes can be classified into the following categories: literary figures, literary works, historical figures and events, geographical information, allusions and references, cultural explanations, and editorial information provided by Sanders himself regarding Shen's personal behavior and experiences. Though all these categories are related to culture to a certain extent, 'cultural explanations' are defined as explanations of specific cultural phenomena and concepts. 
transLogos 2021 Vol 4 Issue 2

Peng, Fangwen, and Linxin Liang, pp. 1-26

An Analysis of the Paratext in Graham Sanders's

Six Records of a Life Adrift: Features and Functions $\underset{\text { A transatation studies ournal }}{\operatorname{tran}}$

(C) Diye Global Communications diye.com.tr|diye@diye.com.tr

The categorization is exclusive, though not strict. To facilitate the research, only each footnote's most relevant function is noted. For detailed information, please see table 1 and figure 1 .

Table 1. Number of footnotes in each record and category

\begin{tabular}{|l|c|c|c|c|c|}
\hline \multicolumn{1}{|c|}{ Category } & Record 1 & Record 2 & Record 3 & Record 4 & Total \\
\hline Literary figures & 12 & 1 & 0 & 10 & 23 \\
\hline Literary works & 7 & 0 & 2 & 2 & 11 \\
\hline Historical figures and events & 2 & 0 & 3 & 10 & 15 \\
\hline Geographical information & 2 & 0 & 1 & 13 & 16 \\
\hline Allusions and references & 5 & 3 & 6 & 15 & 29 \\
\hline Cultural explanation & 11 & 11 & 9 & 16 & 47 \\
\hline Editorial explanation & 1 & 4 & 4 & 1 & 10 \\
\hline \multicolumn{1}{c|}{ Total } & 40 & 19 & 25 & 67 & 151 \\
\hline
\end{tabular}

Figure 1. Proportion of footnotes in each category

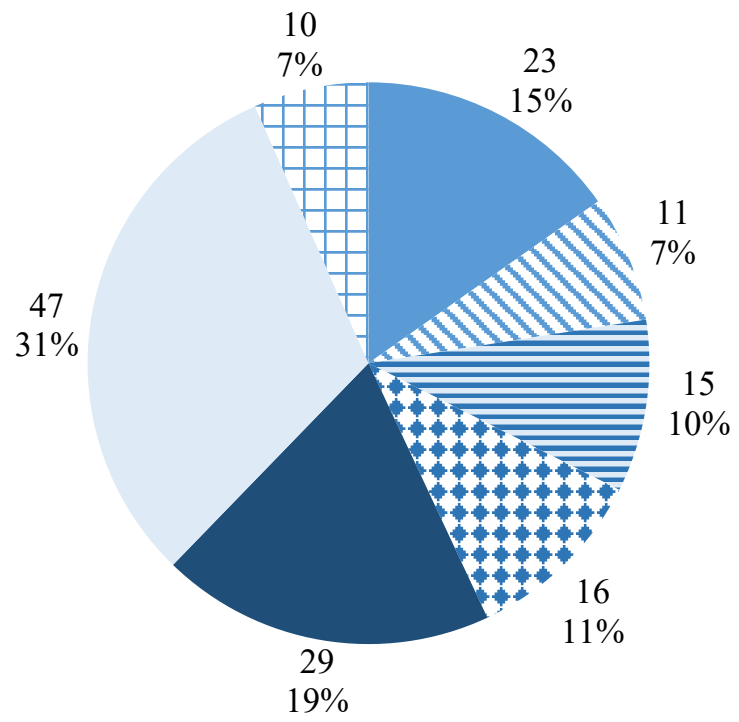

- Literary figures

Literary works

$=$ Historical figures and events

- Geographical information

- Allusions and references

Cultural explanation

- Editorial explanation 
transLogos 2021 Vol 4 Issue 2

Peng, Fangwen, and Linxin Liang, pp. 1-26

$\underset{\text { A Translation Studies Journal }}{\operatorname{tran}}$

An Analysis of the Paratext in Graham Sanders's

Six Records of a Life Adrift: Features and Functions

(c) Diye Global Communications diye.com.tr|diye@diye.com.tr

As can be seen in table 1 and figure 1, most of the footnotes in this version focus on cultural explanations, allusions and references, and literary figures. The following examples better illustrate the features and functions of the footnotes.

\section{Example (1)}

Poetry was used during the Tang dynasty to select men of talent to become officials, but the great masters of poetry were certainly $\mathbf{L i}$ Bai and Du Fu.

\section{Footnote:}

Li Bai (701-702) was the most famous poet in Chinese history. He was known as the Poetry Immortal and was loved for his eccentric and outrageous style, which drew upon fantastic images from history and myth, and for his near constant state of drunkenness. His more staid contemporary, $\mathrm{Du} F u$ (712-770), eventually came to be recognized as the greatest poet in Chinese history. He was called the Poetry Sage or the Poet Historian in later ages and was revered for his technical prowess and moral commitment. (Sanders 2011, 10; emphasis added)

This is the first time Shen references the two great poets (literary figures). Sanders precisely summarizes the poets' most appreciated characteristics and highlights their renown to explain how Chinese people view them and what the poets mean to Shen and his wife.

\section{Example (2)}

Su Shi once traveled here and composed two rhapsodies referring to them as the site where the forces of the $\mathrm{Wu}$ and Wei kingdoms clashed, but he was mistaken about the location. There is dry land at the foot of the cliffs now, but there is still a Two Rhapsodies Pavilion on top.

\section{Footnote:}

Su Shi wrote his pair of "Rhapsodies on Red Cliff" (Chibu fu) (already cited several times by Shen $\mathrm{Fu}$ ) to celebrate outings made with friends to the Red Cliffs at Huangzhou. The pieces are largely philosophical reflections on the ephemerality of life and happiness, but they do evoke the famous battle of Red Cliff on the Yangtze River near the end of the Eastern Han dynasty in $208 \mathrm{CE}$, which ushered in the era of the Three Kingdoms. Even though the actual site of the battle was further upstream, Su Shi's rhapsodies made the red cliffs at Huangzhou equally famous. (Sanders 2011, 129; emphasis added) 
transLogos 2021 Vol 4 Issue 2

Peng, Fangwen, and Linxin Liang, pp. 1-26

This particular footnote explains Su's mistake as pointed out by Shen, presents the Two Rhapsodies and their connection with the Red Cliffs battle, and briefly introduces the historical background of the rhapsodies and their influence. The footnote is categorized under literary works, despite the fact that it includes information about the Red Cliffs as a geological location, Su Shi, and the Three Kingdoms. This is because most importantly, the footnote revolves around the allusion to the Two Rhapsodies, explaining its value and historical influence, so as to illustrate the meaning of Shen's frequent references to it in the narrative. In this record, Shen notes his travels to famous literary and historic destinations, so it is necessary to further reveal his reflections on his travels by making such explanations to the reader. Note that Sanders might have misspelled the original name of the rhapsodies as "Chibu fu," which should be "Chibi fu."

\section{Example (3)}

On August 6,1780, during the Seventh Night Festival of that year, Yun set up a small altar with incense sticks and pieces of melon and fruit in My Choice Hall, where we made our obeisance to the Weaving Girl star.

\section{Footnote:}

Legend has it that the Weaving Girl star (Vega) fell in love with the Herd Boy star (Altair), but the Heavenly Emperor forbade their love and slashed the sky to form a River of Stars (the Milky Way) to keep the lovers separated for eternity. Once a year, on the seventh night of the seventh month in the lunar calendar, magpies took pity on the lovers and flew up into the sky to form a bridge, allowing them to be reunited. Young women would pray to the Weaving Girl star for skills in the domestic arts and to find a good husband. (Sanders 2011, 12; emphasis added)

A deep and thorough explanation of the origin of the festival, the myth behind the celebration, and the worship of the stars offers a distinct perspective for peeking into Chinese culture. Not only does Sanders tell the reader the story of the festival, he also gives the Western names of the stars that are worshipped in Chinese mythology, which can significantly help readers make connections with their own knowledge, probably leading to curiosity-driven discoveries that involve comparing the different images of constellations in the two cultures. Footnotes that provide geological and historical information can have 
transLogos 2021 Vol 4 Issue 2

Peng, Fangwen, and Linxin Liang, pp. 1-26

$\underset{\text { A ransation Studies sournal }}{\operatorname{trans}}$

An Analysis of the Paratext in Graham Sanders's

Six Records of a Life Adrift: Features and Functions

(c) Diye Global Communications diye.com.tr|diye@diye.com.tr

similar functions:

\section{Example (4)}

Her house sat on the site where the palace of the late Yuan dynasty rebel Zhang Shicheng once stood.

\section{Footnote:}

In 1353, the salt merchant Zhang Shicheng (1321-1367) led an uprising called the Red Turban Rebellion against the Mongol rulers of the Yuan dynasty and briefly established his own fiefdom in 1356 with its capital in Suzhou. (Sanders 2011, 23; emphasis added)

\section{Example (5)}

We are actually affiliated with Venerate Tranquility Temple, but the head kitchen only sends us three bushels of dried rice and a jar of salted vegetables once a month.

\section{Footnote:}

Venerate Tranquility Temple (Chongningsi) was established in the early sixth century on the eastern shore of Lake Yangcheng, northeast of Suzhou. (Sanders 2011, 121; emphasis added)

Even if Sanders did not include this information in the footnotes, the reader would have no difficulty understanding the original text because details about a Yuan rebel or a particular temple are not essential for comprehension. However, the footnote explains the peculiarity of the household's location and the historical origin, and better illustrates Shen's footprints throughout his life. Another example features the explanation of the name and title culture in ancient China. It also provides a peek into the intimacy between the couple:

\section{Example (6)}

"I never suspected," I said with a smile, "that Blue Lotus Li would speak to the heart of my Lovely Treasure Chen!"

\section{Footnote:}

Qinglian Jushi (Blue Lotus Hermit) was one of Li Bai's many sobriquets. Shen Fu calls Yun by her own courtesy name here-Shuzhen (Lovely Treasure). (Sanders 2011, 10 ; emphasis added) 
The sobriquet and naming can be quite different in the two cultures. While sobriquets are always supplementary names that describe one's character, in ancient China, they reflected one's life values and literary pursuits. Though Shen is calling Li Bai by his sobriquet here, he is expressing Chen's affection for Li's poetry. Therefore, the great poet's sobriquet now serves to supplement Chen's character, together with her courtesy name. In this way, not only is Chen's literary talent displayed, but so is Shen's affirmation of her with regard to that talent and their romance. Sanders's footnote clarifies the complexity by identifying the titles in this line as a sobriquet and a courtesy name, thus allowing the reader to make the deduction given above.

In the acknowledgments, Sanders states that he will correct errors. The errors he attempts to identify are not confined to those of previous translators but involve the errors of Shen himself as well:

\section{Example (7)}

The best three couplets in five syllables and three in seven syllables would be chosen from among the sixteen couplets.

\section{Footnote:}

Shen $\mathrm{Fu}$ seems to have forgotten that the two people supervising the "examination" would not submit couplets so there should only be twelve couplets in total. (Sanders 2011, 47)

If the details included in this footnote were left out, their absence would not substantially undermine readers' understanding of the original text. Clarifying the ignored uncertainty here, however, can provide a more accurate picture of the Qing literati and the examination system at that time. In addition, the mistake Shen makes here implies that he and his colleagues, when holding this model examination, do not aim to strictly restore the official manner. Their goal is to simply have more opportunities to compose, drink, and socialize. This image corresponds with that created by Sanders in the introduction-Shen as an unsuccessful scholar, who is fond of literature. 
Even though Shen never succeeds at the examination for a political career, his interest and talent in literature cannot be camouflaged or overlooked. In his literary life with Yun, his colleagues, and his friends, great figures and their legacies are the preferred topics, as exemplified in examples 6 and 7. Additionally, living under China's last monarchy, Shen is given the opportunity to appreciate the classics from all the previous dynasties at their best; his allusions and references also range widely throughout literary history. Therefore, it is essential to explain some unfamiliar information to foreign readers, as in example 2.

Cultural differences have been a major obstacle to translation. Modern Chinese culture has seen plenty of that with its Western counterparts, let alone ancient Chinese culture. Sanders thoroughly explains relevant items throughout the text, introducing Chinese festivals and traditions, as in example 3. He also explains homonym concepts (e.g., the name system), clarifies obscurities (e.g., Shen's misunderstandings), and devotes much effort to helping the reader understand Shen's life and world. This is also the reason that he provides corrections or supplements to Shen's story-Sanders, in some parts, links Shen's narration to the content of other records to provide the reader with a more comprehensive view:

\section{Example (8)}

When it comes to my being our uncle's heir, it's true I may have a reduced mourning period because of it, but I never took the least bit of inheritance from him.

\section{Footnote:}

Shen Fu mentions in Record One that he was made the designated heir of his eldest paternal uncle, Shen Sucun, which meant he was responsible for tending his uncle's grave and was permitted a reduced period of mourning for his own father (from three years to one). (Sanders 2011, 77)

The information about family origin that is omitted in Shen's own narration in this record can be found in another, and by adding the information here, Sanders is also interconnecting the segmented impressions of Shen in the reader's mind.

In sum, the layout of these 'discursive' footnotes contributes to the image of the translator by indicating his focuses (i.e., to provide literary and cultural explanations) and 
transLogos 2021 Vol 4 Issue 2

Peng, Fangwen, and Linxin Liang, pp. 1-26

$\underset{\text { A transatation Studies ouvral }}{\operatorname{trans}} \log$

An Analysis of the Paratext in Graham Sanders's

Six Records of a Life Adrift: Features and Functions

(c) Diye Global Communications diye.com.tr|diye@diye.com.tr

offering his understanding of Shen as a romantic indulging in the world of literature and scholarly life.

4.2 The Features and Functions of the Introduction and the Note on the Translation

4.2.1 The Introduction. Sanders's introduction mostly illustrates a picture of Shen's entire life for the reader: his childhood, his family, his romantic relationship, his career and livelihood, his language, and his values. Sanders gives a passionate and candid narration, introducing the book and Shen to the reader.

The introduction starts with an overview of Shen and his renowned piece Fu Sheng Liu Ji. When listing some information about the author, Sanders (2011, viii) adds the adjective "unsuccessful," which is a comment about Shen's life, and it is ubiquitous throughout the introduction. However, Sanders speaks highly of Shen's literary language and praises how this unique trait makes the work so much different from the dramas and novels of his peers in the Ming and Qing dynasties. Sanders points out that Shen's writing, while elegant and concise, provides valuable documents about the society and culture in which he lived in relation to various topics.

\section{Example (1)}

Shen $\mathrm{Fu}$ writes in the concise literary language of poetry, essays, and official histories . . . This choice allows him to slip readily into a poetic lyrical mode of description when depicting his feelings, his relationships, and the beauty of the natural world . . . to provide many vivid details of daily life, describing topics . . . which makes his book a valuable document about the society and culture of late imperial China. (Sanders 2011, viii)

This is a summative statement on Shen's language, applied throughout his narrations. Sanders acknowledges the literary beauty of the work and emphasizes this to the reader in many parts of the introduction. He $(2011, \mathrm{x})$ praises Shen for being "candid" in describing personal failures and praises the records as "powerful" and "genuine." He identifies the literary aesthetics in this way. 
Moreover, Sanders reminds the reader that these records have more often been read as a tragic love story about the author and his wife, Chen Yun, than as the memoir of an insignificant private secretary. Therefore, the reader should be fully aware of what to anticipate in this work: literary aesthetics, simple and beautiful language, a tragic love story, the lifestyle of the late Qing dynasty, and the like.

In most parts of the introduction, Sanders summarizes Shen's life, but before presenting his interpretation of Shen's image, Sanders first provides the reader with background information about the author: geology of the garden city Suzhou, the Shens' family tradition, the author's $\mathrm{Wu}$ dialect, the administration and the occupation of private secretary, etc. For example:

\section{Example (2)}

Shen $\mathrm{Fu}$ even explains some of the unusual Wu-dialect words that he uses in his story, which suggests that he envisioned a readership for his work beyond his close circle of acquaintances. (Sanders 2011, x)

Such detailed examples serve to establish Shen's character in the reader's mind. Shen's explanation of his dialect indicates his literary ambition and suggests that he is a careful person with respect to his own work. By providing similar information in the introduction, Sanders allows the reader to learn about the character in advance. This is also the case for Yun:

\section{Example (3)}

She was orphaned by the death of her father which left her on her own to support her mother and younger brother by doing needle work, even making enough to pay for her brother's schooling. (Sanders 2011, xii)

This set the scene for Sanders to present the main characters. From this, the reader can not only understand the author but also more closely comprehend his records. As quoted in the introduction, Shen, "by his own admission, led an unremarkable, unfulfilled, and itinerant life," and "is remarkably candid about describing his personal failures and portrays" (Sanders 2011, x). Following such a personal admission, Sanders does not shy away from expounding 
transLogos 2021 Vol 4 Issue 2

Peng, Fangwen, and Linxin Liang, pp. 1-26

trans Dogos

A Translation Studies Journal

An Analysis of the Paratext in Graham Sanders's

Six Records of a Life Adrift: Features and Functions

(C) Diye Global Communications diye.com.tr|diye@diye.com.tr

on Shen's failures. He describes how Shen quits his studies and turns to work as a secretary, as his father and brother did, three years after he fails the initial examination. He also hints that Shen's literary talent might be outmatched by Chen's, though the latter, being a woman, has not been properly educated. However, such seeming abasement is how Shen's unique touch is displayed in his life and writings. In Sanders's interpretation, Shen "constantly constructs or encounters small, limited spaces — both physical and in his imagination — where he can feel and express his emotions" (Sanders 2011, xiv). By illustrating the limitations and failures Shen creates and encounters, Sanders is directing the reader's attention to Shen's personal feelings. What he experiences could be termed as frustrating, yet Shen is continuously exhibiting a positive attitude and aiming for his ideal. Though he suffers numerous setbacks, including losing his companion, his beloved wife who shared the same ideas, Shen persists in his pursuit. This is what Sanders wants to tell the reader about Shen.

Apart from Shen's own fate, the introduction elaborates on that of Yun and how the couple's tragic romance unfolds. Sanders quickly gathers the information necessary for the reader to establish Chen's character. Sanders describes how Chen provides for her family when she is young and how she educates herself and becomes literate. He describes how Chen supports Shen in his family life, his career, and on social occasions by maintaining face for her beloved husband, and so on. It is obvious that Sanders thinks highly of this pivotal female character in classic Chinese literature. Weaved into the introduction along with these affirmative narrations are the sacrifices Chen makes for her husband and her family, and her physical, mental, and financial hardships. Sanders successfully introduces Yun to his readers: in his description, Yun is smart, loving, considerate, optimistic, spiritually independent, and willing to sacrifice, all of which are values that are appreciated in both cultures. In this way, Sanders is implying that these records are not only about Shen himself, but are rather a shared record of the couple's married life, which is also a sad one. Therefore, readers will anticipate tragedy and pay more attention to the moods and emotions in the story. It is fair to now conclude that based on Sanders's interpretation of Shen himself and his married life, these 
transLogos 2021 Vol 4 Issue 2

Peng, Fangwen, and Linxin Liang, pp. 1-26

sorrowful emotions are what he intends for readers to appreciate, apart from the social records of the late Qing dynasty.

There are various other notable facts that Sanders mentions. He introduces how the work was initially found in a secondhand bookstore and published in 1877 , and he discusses later publications and translations. He mentions the two missing records that proved to be fanfare. Sanders expresses the belief that the records will be more balanced if viewed with the two missing ones and that the last two records are about how Shen recovers from the loss of his beloved, travels to the Ryukyu Islands, and proposes his ideas about proper methods of living. Secondly, Sanders praises the structure of the record, which mimics the nature of human memory, allowing readers to immerse themselves in the narration.

In the introduction, Sanders expounds on the uniqueness of Fu Sheng Liu Ji: the unique characters of Shen and Yun, their unique life stories, the author's unique language style, the unique social record presented in this work, the unique emotions in Shen's narrative, the unique mode of capturing "a fleeting life of beauty, sorrow, love, and regret" (Sanders 2011, xv), and the unique influence this small book has had on Chinese and Western culture. The introduction extensively illustrates all these aspects that readers need to know about regarding this classic.

In sum, the introduction has some features, including an illustration with plentiful examples and quotes, a summary of some of the essential information, and a discussion about the original text, the translation, and the paratext.

It also has the functions of providing information about the content and the original publication of the work, providing the translator's opinions on literary devices, and the historical and social background of the work, helping the reader establish a general impression of the author and his life, and highlighting other features of the original work.

4.2.2 Note on the Translation. "Note on the Translation" explains essential alterations Sanders made while translating. Here, he points out the additions and changes he made to the previous versions. 
The translator's note is often where the translator stresses her/his or their role, explains her/his or their interpretation of the original work and culture, addresses the differences between the origin and target cultures, and describes the strategy used to translate the cultural references. Through a note on the translation, Sanders discusses some adjustments made in his translation. For instance, in his translation, Sanders converts the units of measurement to the U.S. system and the dates to the Gregorian calendar. He describes the currency and its value at that time, pointing out the Spanish silver dollar that was circulating in China, referencing the exchange rate, and demonstrating the purchasing power of Shen's currency. Sanders uses Pinyin romanization for the names of towns, cities, provinces, and geological scenery, so that it is easier to locate them on maps, which are included in this version. At the same time, Sanders translates the names of streets, gardens, hills, and the like to convey the literal meanings-e.g., the Academy of Cultural Veneration (Chongwen shuyuan) (Sanders 2011, 87). This apparently helps the reader appreciate Shen's preferences in choosing settlement spots. It also resonates with the literary atmosphere of Shen's scholarly community. Sanders thus reveals more essential values of ancient Chinese literature.

In addition, Sanders exemplifies the translation of "wine." He understood that alcoholic drinks were of great importance to Chinese scholars, including Shen's social interactions, and briefly explains how this traditional Chinese drink was made, served, and consumed. He states that though "wine" is a conventional misnomer, he still chose to use this name. Despite the radical difference in ingredients and craftsmanship between Chinese jiu and Western wine, their roles in people's lives were similar and comparable. As mentioned above, one of Sanders's motives to retranslate was to correct the mistakes as much as possible; however, this mission to correct did not include the misnomer "wine," implying that he valued cultural immersion more than literal correctness. "Wine" will continue to be a reminder of the intimacy between Shen and his friends, instead of a nonnative oddity. 
transLogos 2021 Vol 4 Issue 2

Peng, Fangwen, and Linxin Liang, pp. 1-26

Lastly, Sanders addresses other paratextual elements of his translation: the maps, the chronology, the diagram of associates and the family tree, and the list of mentioned historical figures (in addition to the footnotes). He explains the source of illustrations before each record-extracts from The Painting Manual of the Mustard Seed Garden (Jieziyuan huazhuan) - and says that he thought they may have been used by Shen, which is yet another indication that Sanders tries to immerse the reader in Shen's world.

In general, "Note on the Translation" features:

(1) a comprehensive explanation of the adaptation and changes made, and the reasons for these;

(2) a list of added paratextual elements and particular sources.

It aims to:

(1) clarify possible misunderstandings regarding the calendar, weights, measurements, culture, and other social norms;

(2) help immerse the reader in Shen's world;

(3) highlight the translator's emphasis while translating.

\subsection{The Features and Functions of the Title, Illustrations, and Other Elements}

These elements supplement the previous ones in establishing cultural concepts, introducing the characters, and identifying the changes and additions the translator made.

As the first paratextual element encountered by the reader, the front cover communicates the aesthetic, philosophical, and emotional ideas of the work. It is an important part of advertising, as it introduces the work to the market: its readers.

It is necessary to note that the printed cover is a fairly modern creation that dates from the early nineteenth century (Genette 1997). Before modernity, covers were usually muted, providing only information such as the author's name and the title. Now, designing the cover has become a significant task for publishers; the elements that are displayed on covers, alongside the title and the author's name, contain a wealth of information about the publisher, 
transLogos 2021 Vol 4 Issue 2

Peng, Fangwen, and Linxin Liang, pp. 1-26

especially for retranslations.

Figure 2. Front cover of Sanders's version

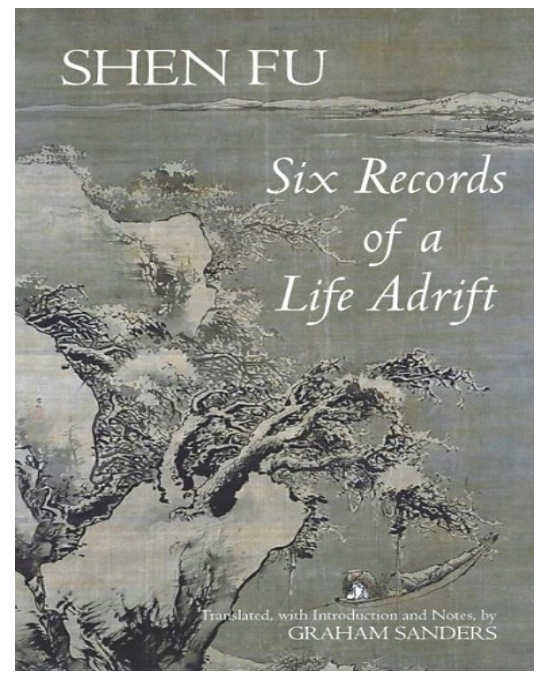

As shown in figure 2, this printed cover, designed by the Hackett Publishing Company, features a background of traditional Chinese ink painting with a lone fisherman fishing and drifting on the water, and winter trees and reefs filling the view. The painting, dominated by a grayish hue, creates a rather desolate atmosphere, which highlights the presence of the fisherman, wearing a straw cape and a rain hat. The image of the fisherman and his boat connects with the titular adjective "adrift," indicating a link between the painting and the story that is told in this work. This demonstrates that emotion plays a significant role in front cover design. Secondly, the archaic Oriental design re-emphasizes that this is a translated Chinese classic, implying that Hackett Classics' target readership is comprised of readers who are drawn to classic Chinese culture. The source of the cover illustration is provided on the back cover: Solitary Angler on a Winter River by Zhu Duan in the Ming dynasty.

The back cover displays comments from various sources, praising Fu Sheng Liu Ji as a classic work of ancient Chinese literature and recognizing Sanders's translation. Below is a short introduction to Graham Sanders as the translator and his occupation as an associate professor of classical Chinese literature at the University of Toronto. These comments equally endorse the author's and translator's work, highlighting the most important feature of this 
transLogos 2021 Vol 4 Issue 2

Peng, Fangwen, and Linxin Liang, pp. 1-26

An Analysis of the Paratext in Graham Sanders's

Six Records of a Life Adrift: Features and Functions trans Dogos

A Translation Studies Journal

(c) Diye Global Communications diye.com.tr|diye@diye.com.tr

work to the reader: a well-refined translation of a beautiful piece of classical Chinese literature, rendered carefully by a qualified professional.

In the Hackett Classics version, the title and the author's name fill the blank spaces. According to Sanders, the title Fu Sheng Liu Ji was derived from a preface that Li Bai wrote for his poem Chunyeyan congdi taohuayuan xu (A spring evening banquet with my cousins in the peach garden) (Sanders 2011, xv). Compared to the previous titles, Six Records of a Floating Life (Pratt and Chiang 1983) and Six Chapters of a Floating Life (Lin 1939), Sanders followed the translation of "records" but proposed a new interpretation of "fu." The definitions from the Collins Online English Dictionary ${ }^{1}$ indicate that while both words describe a state of floating with little or no attachment, the word "adrift" can describe loneliness and a loss of direction, while "floating" is more neutral. This diction choice means that as a translator, Sanders paid more attention to emotions.

As for record titles, Lin, Pratt and Chiang, and Sanders took different approaches to translation. Each version is listed in table 2:

Table 2. Titles of each chapter/record in the three versions

\begin{tabular}{|c|c|c|c|}
\hline Title & Lin's version & $\begin{array}{l}\text { Pratt and Chiang's } \\
\text { version }\end{array}$ & Sanders's version \\
\hline I. Gui Fang Ji Le & Wedded Bliss & $\begin{array}{c}\text { The Joys of the } \\
\text { Wedding Chamber }\end{array}$ & $\begin{array}{l}\text { Delights of } \\
\text { Marriage }\end{array}$ \\
\hline II. Xian Qing Ji Qu & $\begin{array}{c}\text { The Little Pleasures } \\
\text { of Life }\end{array}$ & $\begin{array}{c}\text { The Pleasures of } \\
\text { Leisure }\end{array}$ & Charms of Idleness \\
\hline III. Kan Ke Ji Chou & Sorrow & $\begin{array}{c}\text { The Sorrows of } \\
\text { Misfortune }\end{array}$ & $\begin{array}{l}\text { Sorrows of } \\
\text { Hardship }\end{array}$ \\
\hline IV. Lang You Ji Kuai & The Joys of Travel & $\begin{array}{l}\text { The Delights of } \\
\text { Roaming Afar }\end{array}$ & $\begin{array}{c}\text { Pleasures of } \\
\text { Roaming }\end{array}$ \\
\hline V. Zhong Shan Ji Li & Experience & $\begin{array}{c}\text { A History of Life at } \\
\text { Chungshan }\end{array}$ & $\begin{array}{c}\text { Experiences of } \\
\text { Zhongshan } \\
\end{array}$ \\
\hline VI. Yang Sheng Ji Dao & The Way of Life & The Way of Living & Methods of Living \\
\hline
\end{tabular}

1 Collins Online, s.v. “adrift,” accessed June 16, 2020, https://www.collinsdictionary.com/dictionary/english/adrift. 
As shown in table 2, each version of the translated title portrays a different image of the work's content. Notably, while Lin prioritizes concision, creating a straightforward impression for the reader, foreign translators tend to maintain the original language's regularity. With a strictly symmetric structure of translation, Sanders attempts to balance concision with literary aesthetics. Regarding the diction, Pratt and Chiang's choice caters to the lower contextuality in English-speaking cultures (Peng 2016), making it clear exactly what "sorrow" and "experience" are; this is also the case in Sanders's version. The alteration Sanders decides on and the continuation he follows imply that though he does not explicitly mention his ideas regarding polishing the literary aesthetics in his translation, he attempts this in the details.

Sanders's version also features an expanded appendix, including maps of places where Shen traveled to and worked, a chronology, Shen's list of associates and his family tree, a list of the historical figures Shen mentioned, and an index. Such detailed information can be helpful to scholars and researchers in relevant research.

Finally, in the part of acknowledgments, Sanders (2011, vi) extends his gratitude for "a number of people who made this translation possible," including Shen himself, other scholars and translators, his colleagues, his editors and publishing staff, and his family. Sanders lists the works he consulted: three annotated versions of the original Chinese text, two essays about gender studies, and two overviews of the original work. He also acknowledges the support from many other scholars from China and other countries. It is worth mentioning that Sanders's notes pay extra attention to identify and correct the errors made in the previous versions, expand the annotations, and render the style and tone of the original narration into more modern and colloquial English. These are also the reasons Sanders, together with the Hackett Publishing Company, is proposing a new version of the translation. The community of readers and the academia need a renewed version that accords with modern knowledge and modern English. In general, the part of acknowledgments does not simply list the support the translator had; more helpfully, this part lists the most important 
reference works and explains the intention to propose a retranslation in this era.

In sum, these paratextual elements provide the reader with necessary supplements. Firstly, the translator's goal and efforts in translation are stated in the acknowledgments and implied in the diction of the titles. Sanders concludes the acknowledgments by stating that he attempts to correct previous mistakes, extend the notes, and further polish the translated language to restore authenticity. These three aspects offer guidance to identify and understand his role as a translator.

Secondly, these elements may benefit professional readers more than others. The part of acknowledgments lists the sources the translator consulted, while the maps, the list of associates and the family tree, the list of historical figures, and the index comprise a convenient system for scholars to reference this translation.

Lastly, these elements jointly establish a stage on which the original work in translation is presented. They serve as the framework through which the reader sees the translation and the original work. The covers encourage the reader to enjoy the connotation of the work and beyond. The illustrations suit the original's cultural image and are the visualizations of the verbal story. With these elements, a translated work is hence produced, preparing the reader to venture into the author's world.

\section{Conclusion}

In accordance with Genette's (1991) theory, the paratext in Six Records of a Life Adrift has the following functions: it introduces the translation and the original work to the reader; provides essential supplementary information on the text, especially in the footnotes; and builds a platform on which the translator can establish their image. This case study has proven the value of paratextual analysis in studies on translation and translators. At the same time, it is reasonable to conclude that paratext is indeed a vital part of connecting the original work, the translation, and the target readership, especially for bridging cultural differences and language barriers. 
transLogos 2021 Vol 4 Issue 2

Peng, Fangwen, and Linxin Liang, pp. 1-26

However, this paper can be further improved. The methodology used can be more diversified to grant richer findings. For example, causal-comparative research may be used to establish cause and effect relationships among the translation, publication, and international reception of translated works. This will also encourage new techniques for incorporation into the study of paratext as a theory that is yet to yield more results in various subjects. The nature of paratext (i.e., that it is attached to the text but not incorporated into it) indicates its unique value in explaining and modifying the text itself. Therefore, it can serve as a handy tool in textual analysis.

\section{Acknowledgments}

This paper is based on part of the first author's unpublished thesis submitted to Huazhong University of Science and Technology and is supported by the Humanities and Social Sciences Project, Ministry of Education of the People's Republic of China under Grant No. 18YJC740045. 
transLogos 2021 Vol 4 Issue 2

Peng, Fangwen, and Linxin Liang, pp. 1-26

trans Dogos

A Translation Studies Journal

An Analysis of the Paratext in Graham Sanders's

Six Records of a Life Adrift: Features and Functions

(C) Diye Global Communications diye.com.tr|diye@diye.com.tr

\section{References}

Black, Shirley Marie, trans. 1960. Chapters from a Floating Life: The Autobiography of a Chinese Artist. By Shen Fu. London: Oxford University Press.

Deng, Yan. 2010. "Dazhonghuawenke Ban Fu Sheng Liu Ji Fanyicelue Jiexi." [Analysis of translation strategy of Fu Sheng Liu Ji in library of Chinese classics.] Journal of Anhui University of Technology (Social Sciences) $27 \quad$ (4): 69-71. https://t.cnki.net/kcms/detail?v=3uoqIhG8C46NmWw7YpEsKL-WhGHP2RH_WMv_ DQNmseGEURqkJtk0Z3SSSIwbG7OwZuLWtcY0zZgAqK2AcYxLYXgJJMuxwz4u.

Dong, Hui. 2002. "Lin Yutang Fu Sheng Liu Ji Yingyiben Shangxi." [An analysis of Six Chapters of a Floating Life translated by Lin Yutang.] Journal of Xi'an International Studies $\quad$ University $10 \quad$ (3): $11-15$. https://t.cnki.net/kcms/detail?v=3uoqIhG8C46NmWw7YpEsKL-WhGHP2RH_3D XMT-aCbBhrO_W3Mmhk74EDVzngLwzaFB-pCfnfO2gUFPP4u_iFTWWwJEaqy $\mathrm{xGz}$.

Genette, Gérard. 1991. "Introduction to the Paratext." Translated by Marie Maclean. New Literary History 22 (2): 261-272. doi:10.2307/469037.

1997. Paratexts: Thresholds of Interpretation. Translated by Jane E. Lewin. Cambridge: Cambridge University Press.

Guan, Xingzhong. 2014. "An Alternative Explanation for Translation Variations in Six Chapters of a Floating Life by Lin Yutang." Perspectives 22 (2): 198-221. doi:10.1080/0907676X.2012.722655.

Huang, Peixi. 2018. "Fuwenben Yu Fanyiwenhua Jiangou-Yi Huangdi Neijing Suwen Yingyi Weili." [Paratexts and cultural construction in translation-A case study of English translation of Huangdi Neijing Suwen by Ilza Veith.] Shanghai Journal of Translators, no. 3, 73-79. https://t.cnki.net/kcms/detail?v=3uoqIhG8C46NmWw7YpEsKMypi3qVj28LM4vF8Rafw gpixwXCUWwwzccAzHBSIdxbSNX16QORuuQyI9dQVjVPIDx8R0Wsytps.

Lee, Sang-Bin. 2020. "Reception and Revision of Women-Related Translation Paratexts: South Korean Cases.” The Translator 26 (2): 176-189. doi:10.1080/13556509.2020.1783881. 
transLogos 2021 Vol 4 Issue 2

Peng, Fangwen, and Linxin Liang, pp. 1-26

trans Dogos

A Translation Studies Journal

An Analysis of the Paratext in Graham Sanders's

Six Records of a Life Adrift: Features and Functions

(C) Diye Global Communications diye.com.tr|diye@diye.com.tr

Liang, Linxin, and Xu Mingwu. 2018. "A Comparative Analysis of the Reception of Four English Versions of $\mathrm{Fu}$ Sheng Liu Ji: Translation, Publication and International Circulation.” Translation Review 101 (1): 7-30. doi:10.1080/07374836.2018.1475271.

Lin, Yutang, trans. 1939. Six Chapters of a Floating Life. By Shen Fu. Shanghai: Shanghai Hsi Feng/Westwind Press.

Li, Yuliang. 2005. "Lin Yi Fu Sheng Liu Ji De Deyushi." [Gain and loss of Six Chapters of a Floating Life by Lin Yutang.] Shandong Foreign Language Teaching Journal, no. 6, 79-83. https://t.cnki.net/kcms/detail?v=3uoqIhG8C46NmWw7YpEsKL-WhGHP2RH_OKYKbJw fGDovGYcSqDH10Xh7NhoZmVtlZkMFXOZhU3OESgTGIOE9YWD_i7AR5L8B.

Lu, Fang. 2010. "The Afterlife of Six Chapters of a Floating Life: Three English Translations of Fu Sheng Liu Ji." Translation Review 80 (1): 25-52. doi:10.1080/07374836.2010.10524028.

Luo, Tian, and Zhang Meifang. 2018. "Reconstructing Cultural Identity via Paratexts: A Case Study on Lionel Giles' Translation of The Art of War." Perspectives 26 (4): 593-611. doi:10.1080/0907676X.2017.1401650.

Maher, Brigid. 2016. “'La Dolce Vita' Meets 'the Nature of Evil': The Paratextual Positioning of Italian Crime Fiction in English Translation." The Translator 22 (2): 176-189. doi:10.1080/13556509.2016.1184879.

Peng, Yanhua. 2016. "Fu Sheng Liu Ji Yingyiben Duibi Yanjiu." [Comparative analysis of the English versions of Fu Sheng Liu Ji based on the adaptation and selection theory.] Journal of Zhengzhou University of Aeronautics (Social Science Edition) 35 (2): 114-117. https:/t.cnki.net/kcms/detail?v=3uoqIhG8C46NmWw7YpEsKMypi3qVj28L-63FDqW9 F0hnqUVtDu_jKoFbRqf4XbEIAdg9TM4tRSh8fqpLCaDesJgO-BJfWgMy.

Pratt, Leonard, and Su-hui Chiang, trans. 1983. Six Records of a Floating Life. By Shen Fu. New York: Penguin Classics.

Sanders, Graham, trans. 2011. Six Records of a Life Adrift. By Shen Fu. Indianapolis: Hackett.

Wei, Jiahai. 2017. "Chuci Fanyizhushi De Wenhuagongneng." [The cultural functions of annotations in the English translation of Chu Chi.] Journal of Xi'an International $\begin{array}{llll}\text { Studies } & \text { University } & 25 & \text { (1): }\end{array}$ https://t.cnki.net/kcms/detail?v=3uoqIhG8C46NmWw7YpEsKMypi3qVj28LntHptyn nzpiPCHBHXhEuVar_GqIjOYG0W2boZz81Lsi0ZCZ3E_F165eYDU7QKL9K\&unipl atform $=$ NZKPT. 
transLogos 2021 Vol 4 Issue 2

Peng, Fangwen, and Linxin Liang, pp. 1-26

trans Dogos

An Analysis of the Paratext in Graham Sanders's

A Translation Studies Journal

Six Records of a Life Adrift: Features and Functions

(c) Diye Global Communications diye.com.tr|diye@diye.com.tr

Xiao, Li. 2011. "Fuwenben Zhiyu Fanyiyanjiu De Yiyi." [The significance of paratexts to translation studies.] Shanghai Journal of Translators, no. 4, 17-21. https://t.cnki.net/kcms/detail?v=3uoqIhG8C46NmWw7YpEsKL-WhGHP2RH_EsfUP 6uypmiGaiXJBnKXixMavc7657G06cA8DFZ38HFdcnccnRFfLG_b_a0qjQ92.

Yu, Yanghuan, and Dong Yan. 2020. "Cong Fuwenben Jiedu Cyril Birch Yingyi Mu Dan Ting." [Peripheries of translation: A paratextual interpretation of English translation of Peony Pavilion by Cyril Birch.] Foreign Languages and Their Teaching, no. 5, 127-138.

https://t.cnki.net/kcms/detail?v=3uoqIhG8C46NmWw7YpEsKMypi3qVj28LGACqM pRVR0Cx7F0z4nrArCHMRL-bOxK_9TuEdrrdXuZWRdvfJ2LRGCxfUck0_nDc. 